\title{
Position Location by Time Reversal in Communication Networks
}

\author{
Yuanwei Jin, Nicholas O’Donoughue, and José M. F. Moura \\ Electrical \& Computer Engineering \\ Carnegie Mellon University \\ Pittsburgh, PA 15213
}

\begin{abstract}
Multipath effects are significant in urban or indoor communications. Current position location techniques such as TDOA suffer from multipath effects, which reduces the estimation accuracy. In this paper we propose time-reversal in a wireless communication network, where a mobile terminal wants to determine, through feedback, its own position in the request of the base station. The proposed method improves the estimation accuracy and reduces the estimation variance compared with correlation based method. We derive the closed form of the Cramer-Rao bound (CRB) on the time reversal estimation and the correlation estimation, showing that time reversal achieves a smaller CRB than the correlation method. Numerical examples are presented to illustrate the behavior of these bounds.

Index Terms-Position Measurement, Time Reversal, Cramer-
\end{abstract} Rao Bound, Localization Accuracy

\section{INTRODUCTION}

The ability to locate the source of radio frequency (RF) transmissions has been of considerable interest for many years. Historically, the main interest for position location (PL) has been for military, law-enforcement, and safety applications. More recently, interest has emerged in using position location techniques in a number of applications such as wireless sensor networks (WSN) [1], inventory tracking, indoor geolocation, and emergency 911 (E-911) telephone service [2].

Global Positioning System (GPS) technology is widely used for location-dependent applications. However, this technology has some important limitations: GPS receivers typically do not work indoors or in urban areas where high-rise buildings obstruct the line of sight between the GPS satellite and the receiver. This is the reason why providers of E-911 services have to rely on alternative technologies that use triangulation of information from different base stations. Common techniques used in position location include Time Difference Of Arrival, Angle of arrival (AOA), and Frequency Difference of Arrival (FDOA). All the methods described above suffer from a major disadvantage, namely that the presence of multipath scattering reduces their accuracy. For example, based on the 3GPP urban channel models, we know that the root mean square delay spread in typical dense urban environments can be as much as 10 micro-seconds. This translates into an estimated variance in distance from the base station of 3 kilometers.

In this paper, we develop a time reversal based position location technique to enhance the accuracy of location estimation. Time reversal has been studied recently in the area of electromagnetic transmission [3], [4], [5] and underwater acoustics [6], [7]. For a multipath channel denoted by $h(t)$,

The work is funded by the Defence Advanced Research Projects Agency through the Army Research Office under grant no. W911NF-04-1-0031. the transmitter sends a probe signal $p(t)$, the received signal becomes $s(t)=p(t) * h(t-\tau)$, where $*$ denotes convolution; $\tau$ is the direct path arrival time between the transmitter and the receiver. In time reversal, the transmitted signal is $s_{\text {tr }}(t)=k p(-t) * h(-t)$, where $k$ is the energy normalization scaler. The received signal becomes $y(t)=k(h(-t) * h(t-$ $\tau)) * p(-t)$. Time reversal shortens the multipath channel in that the ( $\tau$-shifted) autocorrelation function $h(-t) * h(t-\tau)$ resembles a Dirac delta function. The richer the multipath scattering, the tighter the time support of the received signal. When a receiver cross-correlates the received signal using a sliding correlator or a matched filter, the distance between the transmitter and the receiver is determined from the arrival time $\tau$ of the correlation peak. For a multipath rich channel, the position location technique using conventional data $s(t)$ suffers from the multipath. However, the time-reversal based position location utilizes data $y(t)$ where the multipath channel is compressed. As a result, time reversal enhances the signal to noise ratio and improves the estimation accuracy.

Time reversal relies on the fact that the transmitter "knows" the channel into which it is transmitting, which requires a feedback mechanism. This is generally true for Time Division Duplex (TDD) networks such as is proposed for the IEEE 802.16 (WiMax) networks. However it is also possible in a Frequency Division Duplex (FDD) network for a transmitter to have Channel State Information (CSI), provided sufficient feedback exists. To implement time reversal, the user terminal must provide feedback to the base station. Let us consider a user terminal that can estimate the forward link channel from the base station to the user. The user terminal then feeds the CSI back to the base station on one of the feedback channels. On reception of the CSI, the base station transmits the timereversed channel response on the first scheduled time slot (for that mobile terminal) in the frame. The mobile receives the time-reversed signal with a certain delay $\tau$ relative to the beginning of the start of the slot. This value of $\tau$ is then used as the estimated distance between the mobile and the base station. Next, we present the conventional correlation based and the time-reversal based time-of-arrival estimation methods.

\section{Conventional Position Location Techniques}

Common location approaches are based on time-of-arrival (TOA), time-difference-of-arrival (TDOA), received signal strength (RSS), and angle-of-arrival (AOA) measurements. In this paper, we consider the TOA method. The TOA method measures the one-way propagation time of the signal travelling between the mobile station and each of the base stations. Synchronization between the mobile terminal and all the base 
stations is important for the one-way propagation measurement process. For two-way ranging, such synchronization may not be necessary. In this paper, we assume that the mobile terminal and the base stations are synchronized.

In conventional matched filtering or correlation-based TOA estimation algorithms, the time at which the matched filter output peaks, or, the time shift of the template signal that produces the maximum correlation with the received signal is used as the TOA estimate. In a narrowband system, however, this value may not be the true TOA since multiple replicas of the transmitted signal, due to multipath propagation, partially overlap and shift the position of the correlation peak. The interested reader can refer to articles on position location techniques and the impact of multipath [8], [9], [10], [11], [12], [13]. For TOA based algorithms, it has been reported that the Cramer-Rao lower bound is affected by the strength of the signal (SNR), integration time, signal bandwidth, and the number of multipath components present. Mitigation of the impairments due to multipath non-line of sight is a key research topic in wireless position location. Next, we derive the analytical expression for the wideband correlation based TOA estimation method and analyze the estimation bias.

We denote the signal emanating from the source (mobile terminal) by $s(t)$. The signal $x(t)$ received at the base station are the attenuated and delayed replicas of the source signal:

$$
x(t)=a_{0} s(t-\tau)+a_{1} s\left(t-\tau-\tau_{d}\right)+v(t)
$$

where $s(t-\tau)$ is the direct path signal; $\tau$ is the true direct path delay; $\tau_{d}$ is the multipath delay. For simplicity, we consider the two-path model here. The two-path model can be extended to the multiple path model and will be studied elsewhere. The process $v(t)$ is the measurement Gaussian noise process with variance $\sigma_{0}^{2}$. For simplicity, we assume that the received signal is normalized so that $a_{0}=1 ; a_{1}$ represents the relative attenuation and the (complex) phase change between the secondary path and the direct path. The cross-correlation method is one of the techniques used to estimate the delay $\tau$ by identifying the first peak of the correlator, i.e.,

$$
\hat{\tau}_{\text {cor }}=\max _{\lambda} \mathfrak{R}\left\{r_{x}(\lambda)\right\}
$$

where $\mathfrak{R}(a)$ is the real part of $a ; r_{x}(\lambda)$ is the cross-correlator output

$$
r_{x}(\lambda)=\frac{1}{T} \int_{0}^{T} x^{*}(t) s(t-\lambda) d t,
$$

where $T$ is the integration time of the correlator. Let $B_{c}$ denote the coherence bandwidth of the channel (or inverse of the delay spread). We also assume that the received signal has been first filtered by an ideal filter of bandwidth $B$. Then, we can express $s(t)$ in terms of its Fourier transform $S(\omega)$ $\left(\omega \in \Omega=\left[-\frac{B}{2}, \frac{B}{2}\right]\right)$ as

$$
s(t)=\int_{-\frac{B}{2}}^{\frac{B}{2}} S(\omega) e^{j \omega t} d \omega .
$$

Hence, if we take the cross-correlation of $s(t)$ and $s(t-\lambda)$, and assume that the correlation interval $T$ is larger than any errors in the delay, we obtain

$$
\begin{aligned}
r_{s}(\lambda) & =\frac{1}{T} \int_{T} s^{*}(t) s(t-\lambda) d t \\
& =\frac{1}{T} \int_{T} d t \int_{\Omega} S^{*}(\omega) e^{-j \omega t} d \omega \int_{\Omega} S\left(\omega^{\prime}\right) e^{j \omega^{\prime}(t-\lambda)} d \omega^{\prime} \\
& =\frac{1}{T} \int_{T} d t e^{-j\left(\omega-\omega^{\prime}\right) t} \int_{\Omega} \int_{\Omega} S^{*}(\omega) S\left(\omega^{\prime}\right) e^{-j \omega^{\prime} \lambda} d \omega d \omega^{\prime} \\
& =\int_{\Omega}|S(\omega)|^{2} e^{-j \omega \lambda} d \omega
\end{aligned}
$$

Using (4) and (5), therefore, we can re-write (3) as follows:

$$
r_{x}(\hat{\tau})=\int_{\Omega}|S(\omega)|^{2} e^{-j \omega \Delta}\left(1+a_{1}^{*} e^{j \omega \tau_{d}}\right) d \omega+\rho_{v},
$$

where the noise term is given by

$$
\rho_{v}=\frac{1}{T} \int_{\Omega} S(\omega) V^{*}(\omega) e^{-j \omega \lambda} d \omega .
$$

$V(\omega)$ is the Fourier transform of the noise $v(t)$. The estimation bias is given by

$$
\Delta=\hat{\tau}_{\text {cor }}-\tau \text {. }
$$

Next, we define $P(\omega)=\frac{|S(\omega)|^{2}}{T}$ as the normalized power spectrum of the signal $s(t)$, where $\int_{-\infty}^{\infty} P(\omega) d \omega=1$. In many practical wireless communication systems, $s(t)$ is a pseudonoise (PN) sequence with the power spectrum $P(\omega)=$ $T_{c} \operatorname{sinc}^{2}\left(\frac{\omega T_{c}}{2}\right)$, where $T_{c}$ is the chip duration of the PN signal. Furthermore, we let

$$
H(\omega)=1+a_{1} e^{-j \omega \tau_{d}}
$$

be the channel frequency response. This channel frequency response has two taps that correspond to the direct path and the second path. Usually, the absolute delay $\tau$ is not included in the channel estimation. We notice that if the delay spread (inverse of the correlation bandwidth of the channel) is larger than the signal waveform duration, then this estimate suffers from a large bias.

\section{TIME REVERSAl Position LocAtion}

In this section, we derive the analytical expression for the time-reversal position location method. Taking the Fourier transform, we re-write (1) as follows:

$$
\begin{aligned}
X(\omega) & =S(\omega) e^{-j \omega \tau}\left(a_{0}+a_{1} e^{-j \omega \tau_{d}}\right)+V(\omega) \\
& =S(\omega) H(\omega) e^{-j \omega \tau}+V(\omega) .
\end{aligned}
$$

In the feedback to the base station, we exclude the absolute delay $\tau$ from the channel frequency response. As stated in section I, after receiving the CSI from the mobile, the base station transmits a waveform that is the time-reversed version of the channel response (equivalently, phase conjugation in the frequency domain) given by

$$
X_{\mathrm{tr}}(\omega)=k H^{*}(\omega) S^{*}(\omega),
$$

where the power normalization factor is given by

$$
k=\sqrt{\frac{\int_{\Omega}|S(\omega)|^{2} d \omega}{\int_{\Omega}|H(\omega) S(\omega)|^{2} d \omega}} .
$$


We note that the proposed time-reversal ranging method is different than the two-way ranging method proposed in [9]. For the two-way ranging method, the re-transmitted probe signals $S(\omega)$ are independent of the multipath channel; while in timereversal ranging, the re-transmitted probe signals $S^{*}(\omega) H^{*}(\omega)$ match with the multipath channel.

Thus, the received signal becomes

$$
\begin{aligned}
Y(\omega) & =X_{\mathrm{tr}} H(\omega) e^{-j \omega \tau}+W(\omega) \\
& =k S^{*}(\omega)|H(\omega)|^{2} e^{-j \omega \tau}+W(\omega),
\end{aligned}
$$

where $W(\omega)$ is the Fourier transform of the Gaussian noise process $w(t)$ of variance $\sigma_{0}^{2}$. Similar to (6), the frequency representation of the cross-correlation of $y(t)$ and $s(t-\lambda)$ becomes

$$
\begin{aligned}
r_{y}(\lambda) & =\int_{\Omega} Y^{*}(\omega) S^{*}(\omega) e^{-j \omega \lambda} d \omega \\
& =\int_{\Omega} k|S(\omega)|^{2}|H(\omega)|^{2} e^{-j \omega(\lambda-\tau)} d \omega+\rho_{w},
\end{aligned}
$$

where the noise term is given by

$$
\rho_{w}=\frac{1}{T} \int_{\Omega} S(\omega) W(\omega) e^{-j \omega \lambda} d \omega .
$$

Hence, the estimate of $\tau$ is obtained by maximizing the real part of the cross-correlation term $r_{y}(\lambda)$, i.e.,

$$
\hat{\tau}_{\text {tr }}=\max _{\lambda} \Re\left\{r_{y}(\lambda)\right\} .
$$

It is straightforward to see that the maximum occurs at $\hat{\tau}_{\text {tr }}=\tau$. This estimate is unbiased, $E\left\{\hat{\tau}_{\text {tr }}\right\}=\tau$.

\section{Performance Analysis}

In this section, we compute the Cramer-Rao bound (CRB) on the error variance of the parameter estimate techniques [14], [15]. We first compute the CRB for the correlation based method, then for the time-reversal based method.

CRB for correlation method: In our analysis, we assume that the only unknown parameter is $\tau$, and that we have perfect knowledge of the deterministic values $a_{1}$ and $\tau_{d}$. Let $\mathbf{x}=[x(0), \ldots, x(N-1)]^{T}$ be the vector of $N$ sample values of $x(t)$. The unknown parameter $\tau$ needs to be estimated from the information provided by the measurement vector $\mathbf{x}$. Denote the conditional probability density function of the measurements by $p(\mathbf{x} \mid \tau)$. Then the Fisher information is given by $J(\tau)=-E\left\{\frac{\partial^{2} \log p(\mathbf{x} \mid \tau)}{\partial \tau}\right\}$. Given $N$ discrete samples of $\mathbf{x}$, we can compute the discrete joint probability density function. However, here, we will consider the continuous time equivalent of the probability density function $p(\mathbf{x} \mid \tau)$ (see, e.g. [15] for the discussion where the number of dimensions of $\mathbf{x}$ tends to infinity). The log-likehood function of $p(\mathbf{x} \mid \tau)$ can be written as

$$
\begin{gathered}
\log p(\mathbf{x} \mid \tau)=\frac{1}{\sigma_{x}^{2}} \int_{T}\left|x(t)-\left[s(t-\tau)+a_{1} s\left(t-\tau-\tau_{d}\right)\right]\right|^{2} d t \\
=\frac{1}{2 B \sigma_{x}^{2}} \int_{T}\left|X(\omega)-S(\omega) e^{-\jmath \omega \tau}\left(1+a_{1} e^{-\jmath \omega \tau_{d}}\right)\right|^{2} d \omega .(19)
\end{gathered}
$$

Here, we drop the terms that do not contribute to the Fisher information statistic, and apply the Parseval's theorem to evaluate the expression in the frequency domain. We define $\sigma_{x}^{2}$ as the variance of the function $x(t)$ :

$$
\sigma_{x}^{2}=\left(1+a_{1}^{2}\right) \sigma_{s}^{2}+\sigma_{n}^{2} .
$$

Evaluating the second partial derivative with respect to $\tau$ and computing the Fisher information statistic [14], [15] yield

$$
\begin{aligned}
J_{\text {cor }}(\tau)= & \frac{1}{B \sigma_{x}^{2}} \int_{\Omega} \omega^{2}|S(\omega)|^{2}\left(1+a_{1}^{2}+2 a_{1} \cos \left(\omega \tau_{d}\right)\right) d \omega \\
= & \frac{1}{B \sigma_{x}^{2}}\left[\left(1+a_{1}^{2}\right) \int_{\Omega} \omega^{2}|S(\omega)|^{2} d \omega\right. \\
& \left.+2 a_{1} \int_{\Omega} \omega^{2}|S(\omega)|^{2} \cos \left(\omega \tau_{d}\right) d \omega\right] .
\end{aligned}
$$

We define the following two quantities

$$
\begin{aligned}
\beta^{2} & =\int_{\Omega} \omega^{2}|S(\omega)|^{2} d \omega \\
\beta_{\lambda}^{2} & =\int_{\Omega} \omega^{2}|S(\omega)|^{2} \cos (\omega \lambda) d \omega
\end{aligned}
$$

which leads to the CRB on our time delay estimate:

$$
\mathrm{CRB}_{\text {cor }}=J_{\text {cor }}^{-1}(\tau)=\frac{B \sigma_{x}^{2}}{\left(1+\left|a_{1}\right|^{2}\right) \beta^{2}+2 a_{1} \beta_{\tau_{d}}^{2}} .
$$

The CRB derived here assumes an unbiased estimator. In our case, however, we assume that the multipath is sufficient to cause a bias in the estimate. The CRB for the biased estimator is given by

$$
\operatorname{Var}\{\hat{\tau}\} \geq\left(\frac{\partial E[\hat{\tau}]}{\partial \tau}\right)^{2} \mathrm{CRB}_{\text {cor }}
$$

Note that, since $\hat{\tau}=\tau+\Delta$ and $\tau$ is deterministic, $\operatorname{Var}\{\hat{\tau}\}=$ $\operatorname{Var}\{\Delta\}$. Thus, $\operatorname{Var}\{\Delta\} \geq\left(\frac{\partial E[\Delta]}{\partial \tau}\right)^{2} \mathrm{CRB}_{\text {cor }}$.

CRB for TR method: Let $\mathbf{y}=[y(0), \ldots, y(N-1)]^{T}$ be the vector of $N$ sample values of $y(t)$, where $y(t)$ is the time domain representation of $Y(\omega)$. Taking the loglikelihood function of $p(\mathbf{y} \mid \tau)$, and again, using the equivalent continuous time of the probability density function [15] and by the Parseval's theorem, we obtain

$$
\log p(\mathbf{y} \mid \tau)=\frac{1}{B \sigma_{y}^{2}} \int_{\Omega}|Y(\omega)-Z(\omega)|^{2},
$$

where $Z(\omega)$ is the Fourier transform of $z(t)$

$$
\begin{aligned}
Z(\omega) & =X_{\mathrm{tr}}(\omega) e^{-\jmath \omega \tau}\left(1+a_{1} e^{-\jmath \omega \tau_{d}}\right) \\
& =k S^{*}(\omega) e^{-\jmath \omega \tau}\left|1+a_{1} e^{-\jmath \omega \tau_{d}}\right|^{2},
\end{aligned}
$$

and

$$
\sigma_{y}^{2}=\left(1+2 a_{1}+a_{1}^{2}\right) \sigma_{s}^{2}+\sigma_{n}^{2}
$$

Evaluating the partial derivatives yields:

$$
\begin{aligned}
J_{\mathrm{tr}}(\tau)= & \frac{k^{2}}{B \sigma_{y}^{2}}\left[\left(1+4\left|a_{1}\right|^{2}+\left|a_{1}\right|^{4}\right) \int_{\Omega} \omega^{2}|S(\omega)|^{2} d \omega\right. \\
& +2 a_{1}\left(1+\left|a_{1}\right|^{2}\right) \int_{\Omega} \omega^{2}|S(\omega)|^{2} \cos \left(\omega \tau_{d}\right) d \omega \\
& \left.+2\left|a_{1}\right|^{2} \int_{\Omega} \omega^{2}|S(\omega)|^{2} \cos \left(2 \omega \tau_{d}\right) d \omega\right] .
\end{aligned}
$$




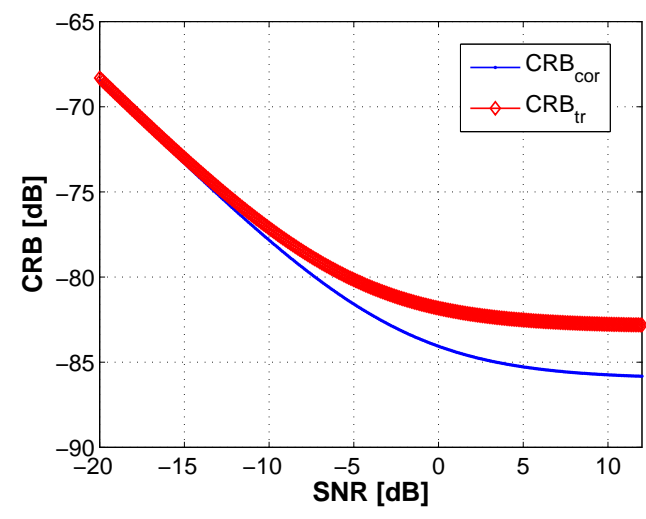

Fig. 1. Cramer-Rao bound vs. SNR for TR and correlation methods.

This leads to the CRB on our time reversal based time delay estimate:

$$
\begin{aligned}
\mathrm{CRB}_{\mathrm{tr}} & =J_{\mathrm{tr}}^{-1}(\tau) \\
& =\frac{B \sigma_{y}^{2} / k^{2}}{\left(1+4 a_{1}^{2}+a_{1}^{4}\right) \beta^{2}+2 a_{1}\left(1+a_{1}^{2}\right) \beta_{\tau_{d}}^{2}+2 a_{1}^{2} \beta_{2 \tau_{d}}^{2}} .
\end{aligned}
$$

It is straightforward to see that $\mathrm{CRB}_{\text {tr }}=\mathrm{CRB}_{\text {cor }}$ if the secondary multipath does not exist, i.e., $a_{1}=0$ (note that, in this case, the normalization factor reduces to $k=1$ ).

\section{Numerical Simulations}

To gain insight into the behavior of the bounds for the proposed time-reversal scheme, we carry out numerical simulation studies. The SNR is defined as $\frac{\sigma_{s}^{2}}{\sigma_{n}^{2}}$, where $B$ is the signal bandwidth. The propagation paths are assumed to include a direct path and a secondary path characterized by $a_{1} e^{-j \omega \tau_{d}}$ where $\tau_{d}$ is the relative delay of the second path. The delay spread $\tau_{\mathrm{sp}}=B_{c}^{-1}$ is chosen to be $1 \mu \mathrm{s}$, where $B_{c}$ is the coherence bandwidth. We vary the $a_{1}$ and $\tau_{d}$ of the multipath. Fig. 1 depicts the CRB for the TR method and the correlation method when $a_{1}=0.9$ and $\tau_{d}=1 \mu \mathrm{s}$. The CRB for the TR method is smaller than the correlation method CRB as we vary the SNR. We define the CRB ratio as $\eta=\frac{\mathrm{CRB}_{\text {tr }}}{\mathrm{CRB}_{\text {cor }}} \geq 1$. Fig. 2 depicts the CRB ratio vs. $a_{1}$ at different relative multipath delays. The results show that $\eta$ increases as the amplitude of the second path $a_{1}$ increases to 1 , indicating a gradually stronger second path. When $a_{1}=0$, the ratio becomes, as expected, 1 . This is caused by both the normalization factor $k$ and the strength of the multipath $a_{1}$. At a fixed $a_{1}$, the ratio increases as the relative delay $\tau_{d}$ is decreased because the second path is closer to the direct path, yielding more gain for the TR method. This gain is expected to saturate with a single dominant path.

\section{Vi. CONClusion And Future Work}

In this paper, we develop a time reversal based position location method in a wireless communication system. We show that matching the transmitted signal to the multipath propagation channel by time reversal improves the delay estimation accuracy and reduces the estimation variance compared with the cross-correlation based method. The time-reversal step requires feedbacks between the mobile terminal and the

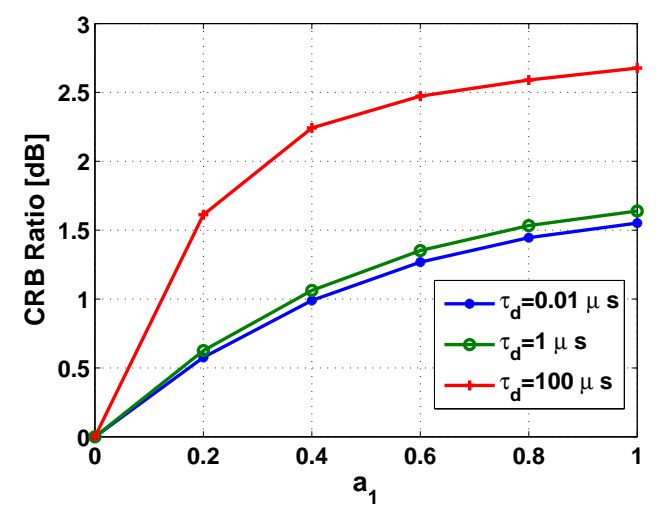

Fig. 2. $\mathrm{CRB}$ ratio $\eta$ in $\mathrm{dB}$ with varying $a_{1}$ and relative delay $\tau_{d}$.

base station. This paper studies the ideal case where the timereversed waveform matches the propagation channel perfectly. In reality, channel estimation error reduces the accuracy of the delay estimate (see, e.g. [16] for the influence of noise on detection performance). In future research we will study the impact of error or channel mismatch on the estimation performance.

\section{REFERENCES}

[1] S. Gezici, Z. Tian, G. B. Giannakis, H. Kobayashi, A. F. Molisch, H. V. Poor, and Z. Sahinoglu, "Localization via ultrawideband radios," IEEE Signal Processing Magazine, pp. 70-84, July 2005.

[2] "FCC adopts rules to implement enhanced 911 for wireless services," FCC News, CC docket, no. 94-102, June 121996.

[3] Y. Jin, Y. Jiang, and J. M. F. Moura, "Multiple antenna time reversal transmission in ultra-wideband communications," in Proc. of IEEE Globecom. IEEE, November 2007.

[4] J. M. F. Moura and Y. Jin, "Detection by time reversal: single antenna," IEEE Transactions on Signal Processing, vol. 51, no. 1, pp. 187-201, January 2007.

[5] D. D. Stancil, A. G. Cepni, B. E. Henty, Y. Jiang, Y. Jin, J.-G. Zhu, and J. M. F. Moura, "Super-resolution focusing and nulling in rich multipath environments using time-reversal techniques," in Intl' Conf. on Electromagnetics in Advanced Applications (ICEAA). IEEE, April 2005.

[6] M. Fink, "Time reversed acoustics," Physics Today, vol. 50, no. 3, pp. 34-40, 1997.

[7] D. G. Albert, L. Liu, and M. Moran, "Time reversal processing for source location in an urban environment," J. Acoust. Soc. Am., vol. 118, no. 2, pp. 616-619, August 2005.

[8] C. Drane, "Positioning GSM telephones," IEEE Communications Magazine, pp. 46-59, April 1998.

[9] J. Y. Lee and R. A. Scholtz, "Ranging in a dense multipath environment using a UWB radio link," IEEE Journal on Selected Areas in Communications, vol. 20, no. 9, pp. 1677-1683, December 2002.

[10] Y. Qi, H. Kobayashi, and H. Suda, "On time of arrival positioning in a multipath environment," IEEE Transactions on Vehicular Technology, vol. 55, no. 5, pp. 1516-1526, September 2006.

[11] M. J. D. Rendas and J. M. F. Moura, "Cramer-Rao bounds for location systems in multipath environments," IEEE Transactions on Signal Processing, vol. 39, no. 12, pp. 2593-2610, December 1991.

[12] B. Friedlander, "Accuracy of source localization using multipath delays," IEEE Transactions on Aerospace and Electronic Systems, vol. 24, no. 4, pp. 346-359, July 1988.

[13] J. Iannello, "High resolution multipath time delay estimation for broadband random signals," IEEE Transactions on Acoustics, Speech, and Signal Processing, vol. 36, no. 3, pp. 320-327, March 1988.

[14] L. L. Scharf, Statistical Signal Processing: Detection, Estimation, and Time Series Analysis. Reading, MA: Addison-Wesley Publishing Company, 1991.

[15] H. L. VanTrees, Detection, Estimation, and Modulation Theory: Part I. New York, NY: John-Wiley \& Sons, Inc., 1968.

[16] Y. Jin and J. M. F. Moura, "Asymptotic noise analysis in time reversal detection," in Proc. 40th Asilomar Conf. Signals, Syst., Comp., Pacific Grove, CA, November 2006, pp. 772-776. 